Revue des patrimoines

\title{
À propos de la restitution en ethnologie. Compte rendu du deuxième atelier des ethnopôles
}

Carcassonne, GARAE, 13 et 14 juin 2013

\section{Christian Hottin}

\section{OpenEdition}

\section{Journals}

Édition électronique

URL : http://journals.openedition.org/insitu/10528

DOI : $10.4000 /$ insitu. 10528

ISSN : 1630-7305

Éditeur

Ministère de la Culture

Référence électronique

Christian Hottin, «À propos de la restitution en ethnologie. Compte rendu du deuxième atelier des ethnopôles », In Situ [En ligne], 21 | 2013, mis en ligne le 04 septembre 2013, consulté le 22 septembre 2020. URL : http://journals.openedition.org/insitu/10528 ; DOI : https://doi.org/10.4000/insitu.10528

Ce document a été généré automatiquement le 22 septembre 2020.

\section{c) (i) $\odot \odot$}

In Situ Revues des patrimoines est mis à disposition selon les termes de la licence Creative Commons Attribution - Pas d'Utilisation Commerciale - Pas de Modification 4.0 International. 


\section{À propos de la restitution en ethnologie. Compte rendu du deuxième atelier des ethnopôles}

Carcassonne, GARAE, 13 et 14 juin 2013

\section{Christian Hottin}

1 Après le premier séminaire, tenu à la ferme Courbet de Flagey en octobre 2012 et consacré à la question de la construction des territoires, le deuxième atelier des ethnopôles s'est déroulé les 13 et 14 juin 2013 dans les locaux du GARAE (groupe audois de recherche et d'animation ethnographique). Il avait pour thème la restitution des enquêtes ethnologiques.

Quatre structures détiennent aujourd'hui le label «ethnopôle», délivré par le ministère de la Culture et de la Communication (direction générale des patrimoines, département du pilotage de la recherche et de la politique scientifique) : le GARAE (Aude), la Maison du Fleuve Rhône (Rhône), le musée conservatoire de Salagon (Alpesde-Haute-Provence) et l'ethnopôle « Pays de Courbet, pays d'artiste » (Musée Courbet, Doubs). Chaque ethnopôle s'engage, dans le cadre de sa convention de partenariat avec le ministère, à mener conjointement des activités de recherche, selon les principes de la recherche scientifique, et des actions culturelles ancrées dans le territoire où est implanté l'ethnopôle. Cette situation particulière explique aisément pour quelle raison la question de la restitution des enquêtes ethnologiques est particulièrement pertinente pour les ethnologues de ces musées ou associations.

3 Trois études de cas ont été présentées lors de l'atelier et de nombreuses mises en parallèles ont été faites avec d'autres expériences. Une mise en perspective historique et épistémologique s'imposait toutefois. Elle a été proposée par Daniel Fabre en ouverture de ces journées. Dans les décennies qui suivent la dernière guerre, alors que se développe en France une ethnologie du proche, la question de la réception de leurs travaux ne préoccupe guère les ethnologues, et, plus généralement, les chercheurs en sciences sociales : la conception qui domine reste celle d'une séparation étanche entre les informateurs du scientifique, qui par définition ne liront pas les analyses tirées de 
l'observation, et le monde académique, seul destinataire de celles-ci. Plusieurs affaires, au cours des années 1960 et 1970, montrent toutefois que cette posture expose le chercheur à des déconvenues et que le "terrain» est susceptible de réaction d'une vivacité déroutante: Edgar Morin avec les Bretons de Plozevet (que le bourg soit rebaptisé Plodémet dans le livre issu de l'enquête ne changera rien à l'émotion soulevée par le tableau dressé de la jeune locale ${ }^{1}$ ), Yvonne Verdier avec les femmes de $\operatorname{Minot}^{23}$ en font l'expérience. Pour cette dernière, le conflit avec l'informatrice se poursuit en procès. La plaignante est déboutée et le jugement caractérise de manière intéressante le travail de l'ethnologue en reconnaissant sa capacité à se protéger en métamorphosant la société étudiée en une société anonyme: les personnages rencontrés sont des «types » construits par mélange des observations faites auprès de différentes personnes, les noms sont modifiés. Quelques années plus tard, l'issue d'une autre affaire est tout autre : se reconnaissant sur un cliché ancien sélectionné par une association bretonne dans le cadre d'une exposition sur le mariage, un particulier porte l'affaire devant les tribunaux, perd en première instance, mais gagne en appel. À la même époque, d'autres expériences sont pourtant déjà en cours, qui contribuent à éviter ces confrontations : la restitution de l'enquête auprès du terrain apparaît de plus en plus comme un impératif moral. Dans certains cas, comme pour les enquêtes orales de l'historien Philippe Joutard dans les Cévennes, une forme de contractualisation avec les détenteurs locaux de la mémoire protestante est même la condition sine qua non de la réussite de l'enquête. Dans le champ de l'ethnologie, la collection Terre d'Aude, avec les ouvrages publiés par Claudine Fabre-Vassas, Christiane Amiel et Jiordana Charruti, témoigne d'un travail de terrain qui laisse une large place à l'expression directe des personnes rencontrées, qui ne sont plus de simples informateurs, mais accèdent progressivement à la stature de témoin, en attendant de devenir de véritables figures : femmes de la vigne ${ }^{4}$, femmes du pays de Sault ${ }^{5}$, conteurs (tel l'illustre Pierre Pous $\left.{ }^{6}\right) \ldots$

Qu'en est-il des formes actuelles de la restitution des enquêtes? Quels sont les méthodes et les médias employés par les ethnologues? Quelles sont les réactions suscitées par celles-ci ? Quelle est l'attitude des commanditaires?

5 Un premier ensemble de réponses est apporté par Sylvie Sagnes et Véronique Moulinié à travers la présentation de leurs enquêtes sur la mémoire de la Retirada dans le midi de la France, travail réalisé dans le cadre d'une commande du conseil régional de Languedoc-Roussillon: une exposition, un livre largement illustré ${ }^{7}$, un film ${ }^{8}$, des conférences et des projections suivies de débats ont été les différentes formes de restitutions de ces enquêtes. Un constat s'impose: reposant sur une méthodologie parfois méconnue des commanditaires, le travail de l'ethnologue et ses résultats déroutent parfois le commanditaire lui-même. Loin de mettre en forme un ensemble de témoignages bruts pour proposer une histoire conventionnelle de la retraite des Républicains espagnols, l'enquête ethnologique fait émerger des aspérités de la mémoire, des points de fixation, des déplacements du centre de gravité de la mémoire (de la figure du combattant pour la première génération vers celle de la victime pour les générations suivantes) et parfois même un désintérêt marqué pour certains sujets (à l'image de celui exprimé par ces enfants nés à la maternité d'Elne, un lieu dont l'importance ne cesse de croître dans les commémorations officielles, puisqu'il offre une alternative positive et valorisante à la mémoire des camps du littoral, nécessairement douloureuse). L'ouvrage publié, largement diffusé, présente l'avantage d'offrir un support à la mémoire, en rassemblant des œuvres d'art réalisées dans les camps, alors que ces derniers ont laissé assez peu de traces matérielles in situ. Il crée 
surtout un condensé de mémoire qui favorise sa réactivation : certaines œuvres, qui ne suscitaient chez leur possesseur qu'un discours neutre et distancié, provoquent un afflux d'émotions lorsqu'elles réapparaissent dans le contexte du livre ou d'une exposition. Le film, quant à lui, rencontre un très grand succès, libérant la parole à la suite de projections et ouvrant la voie à d'autres récits de mémoire, par exemple sur la vie dans l'Espagne franquiste.

6 L'enquête de Jean-Pierre Piniès et Christiane Amiel s'inscrit quant à elle dans une série de travaux qui questionnent l'histoire de lieux au devenir complexe: après la chartreuse de Villeneuve-lès-Avignon ${ }^{9}$ et avant, peut-être, la prison d'Alès, ils ont tenté de radiographier, à la demande d'une communauté de communes, le passé de l'Abbaye d'Aniane, qui devint d'abord une colonie pénitentiaire avant d'être transformée en institut d'éducation spécialisée. Lieu de surveillance, de punition et de redressement, sujet à la violence continue exercée par l'institution et à celle, sporadique et spectaculaire, des pensionnaires. Certaines strates de cette mémoire, les plus récentes notamment, demeurent douloureuses, difficiles à percer. En revanche, à l'occasion des restitutions auprès du commanditaire ou d'un bistrot des ethnologues, il est possible de débattre de l'époque la plus ancienne, celle de la colonie pénitentiaire, active jusque dans les années 1940. Ces rencontres rendent possible l'acceptation de ce passé, sa démystification, et ouvrent, peut-être, la voie à un possible travail de mémoire sur la période la plus récente.

7 Avec le troisième exemple proposé, celui de l'opération des Archives du sensible ${ }^{10}$, mené par Marion Tiba dans le cadre du Parc Naturel Régional de la Narbonnaise, on quitte le domaine de la restitution, somme toute assez classique, de l'enquête ethnologique auprès du terrain. Dans ce projet multiforme, aux développements continus, les lignes de fractures traditionnelles s'estompent : le comité d'experts passe au second plan, tandis que s'affirme la figure des "passeurs du territoire ", les acteurs locaux s'initient à la recherche et la pratiquent, tandis que les chercheurs s'impliquent très directement dans l'action culturelle. Le projet se nourrit de sa dynamique propre et s'enrichit continuellement de nouvelles rubriques, rendues possibles par la plasticité qu'offre le travail sur Internet. Il n'est plus seulement question ici d'enquête et de restitution, mais de portraits, de tableaux, de films, d'œuvres d'art.

L'ethnopôle et le Parc Naturel Régional, à travers le projet de site des Archives du sensible, offrent un cadre structurant pour répondre aux demandes multiples de patrimoine et de mémoire exprimées par les élus du parc et par sa population. Sur ce territoire qui n'est pas doté de toutes les caractéristiques qui répondent à la définition usuelle du parc naturel régional (notamment l'unité des paysages), c'est la culture qui permet de tisser les liens nécessaires à la construction du territoire.

9 Pour en résumer rapidement le principe, les Archives du sensible sont un projet, ouvert et continu, de production d'archives contemporaines ${ }^{11}$, sensibles, vivantes, sur le PNR de la Narbonnaise : ces archives consistent, au départ, en enquêtes sur le territoire et ses habitants, accompagnées de formation aux techniques d'investigation ethnographiques ou historiques ${ }^{12}$, et suivies de restitutions de celles-ci sous la forme de publications ${ }^{13}$, d'expositions et de veillées; l'ensemble de ces productions vient à son tour enrichir le site, qui connaît sans cesse de nouveaux développements (par exemple à travers la création d'œuvres graphiques, photographiques, littéraires ${ }^{14}$ ou plastiques). L'équipe scientifique du projet, animée par Marion Tiba revendique un triple postulat méthodologique : affirmer le primat de la démarche sur le contenu, assumer le fait 
d'être à la fois juge et partie, renouveler les formes du travail collectif tout en éradiquant les clichés. Un pari qui semble largement tenu aujourd'hui, et qui pourrait être un jour complété par la numérisation et la mise en ligne de toutes les archives du projet, offrant de la sorte au visiteur des clefs d'interprétation de celui-ci.

La table ronde conclusive portait sur les nouveaux informateurs et nouveaux énonciateurs de l'ethnologie: ce fut l'occasion de revenir sur l'expérience particulièrement riche des Archives du sensible, cet espace virtuel, superposé au territoire du parc où se croisent acteurs du terrain, chercheurs, et créateurs. Il y a là, à n'en pas douter, une piste de réflexion plus générale pour l'anthropologie. Où qu'il aille de nos jours l'anthropologue ne rencontre plus pour terrain d'études de milieu pur de toute atteinte extérieure, où s'exprimerait spontanément, à travers la vie de tous les jours et les rituels un «inconscient culturel», il ne vit plus cette situation privilégiée qui lui permettait d'en expliciter les processus. Partout où il va, il rencontre des sociétés qui connaissent ce qui constitue leur ciment, et qu'elles désignent sous le nom de culture.

\section{NOTES}

1. - MORIN, Edgar. Commune en France : la métamorphose de Plodémet. Paris : Fayard, 1967, 288 p.

2. - VERDIER, Yvonne. Façons de dire, façons de faire : la laveuse, la couturière, la cuisinière. Paris : Gallimard, 1979, [24]-347 p.

3. - ZONABEND, Françoise. «Retour sur archives: ou comment Minot s'est écrit ». L'Homme, 2011/4, n² 200, p. 113-140.

4. - AMIEL, Christiane, CHARUTY, Giordana et FABRE-VASSAS, Claudine. Jours de vigne : Les femmes dans les pays viticoles racontent le travail. Villelongue d'Aude: Atelier du Gué, 1981, 143 p. (collection Terre d'Aude, 9).

5. - CHARUTY, Giordana, FABRE-VASSAS, Claudine, FINE, Agnès. Gestes d'amont : les femmes du pays de Sault racontent le travail. Villelongue d'Aude: Atelier du Gué, 1980, 125 p. (collection Terre d'Aude, 7).

6. - POUS, Pierre. Cinq contes populaires du pays de Sault (édition établie et présentée par Daniel Fabre). Villelongue d'Aude : Atelier du Gué, 1979, 107 p. (collection Terre d'Aude, 2).

7. - MOULINIÉ, Véronique. La Retirada: mots et images d'un exode. Carcassonne : Garae-Hésiode, 2009, $214 \mathrm{p}$.

8. - CHEVAIS, Marie (réal.), MOULINIÉ, Véronique et SAGNES, Sylvie (aut.). Mémoires de la Retirada. Meudon : CNRS Images, 2012, DVD Vidéo, 35 min.

9. - PINIÈS, Jean-Pierre. La chartreuse de Villeneuve-lès-Avignon: métamorphoses d'un monument. Marseille et Villeneuve-lès-Avignon : Laffitte et La Chartreuse, 2010, 379-XVI p.

10. - Voir : http://www.parc-naturel-narbonnaise.fr/archives_du_sensible/.

11. - Les Archives départementales de l'Aude sont partenaires de ce projet.

12. - À titre d'exemple, la collecte et la numérisation de papiers privés, ou la mise en ligne de films des années 1950 montrant la vie locale, sont accompagnées de textes de la directrice des Archives départementales donnant des conseils méthodologiques pour écrire l'histoire d'une commune. 
13. - Voir, par exemple, «La Nadière: l'île paradoxale»: http://www.parc-naturelnarbonnaise.fr/archives_du_sensible/sensible/carnets/la_nadiere/nadiere.html.

14. - Voir, par exemple le texte de Christophe Pradeau, «Belle lurette»: http://www.parcnaturel-narbonnaise.fr/archives_du_sensible/sensible/la\%20place/ecrites/pradeau/

pradeau.html.

\section{AUTEUR}

\section{CHRISTIAN HOTTIN}

Département du pilotage de la recherche et de la politique scientifique, Direction générale des patrimoines, Ministère de la Culture et de la Communication christian.hottin@culture.gouv.fr 